\title{
INFLUENCE DES CONDITIONS DE NUTRITION MINÉRALE SUR LA CROISSANCE DE L'ÉPICÉA COMMUN SUR LES PLATEAUX CALCAIRES DE L'EST DE LA FRANCE
}

\author{
F. LE TACON et C. MILLIER
}

avec la collaboration technique de Maryse BItsch, D. Bouchard, Françoise Georges, Danielle SALmon et Régine RIELA

Station de Recherches sur les Sols forestiers et la Fertilisation,

Station de Biométrie,

Centre national de Recherches forestières, 54 - Nancy

Institut national de la Recherche agronomique

\section{RESUME}

La croissance de l'épicéa sur les plateaux calcaires de l'est de la France est très liée aux conditions d'alimentation minérale. L'azote apparaît être l'élément le plus important. La production plus élevée observée en sol décarbonaté s'explique en partie par une meilleure nutrition azotée ; d'autre part, les variations de production observées en sols carbonatés sont très liées aux possibilités d'alimentation en azote.

La chute de production observée en sol carbonaté peut également être en partie attribuée à une déficience en manganèse. La nutrition en phosphore joue aussi un rôle important surtout en sol carbonaté. Un niveau de nutrition élevé en phosphore peut permettre de compenser en partie un excès de calcaire.

L'influence de la nutrition en calcium sur la croissance est difficile à mettre en évidence car l'augmentation de la disponibilité en calcium ou en calcaire du milieu se répercute surtout sur la nutrition en manganèse et en azote, ce qui masque l'action propre du calcium au niveau des aiguilles.

Le niveau de nutrition en potassium, magnésium et fer ne contribue que très peu à expliquer les variations de production de l'épicéa. 


\section{INTRODUCTION}

Dans deux études précédentes, nous avons abordé le problème du comportement de l'épicéa sur les plateaux calcaires de l'est de la France. Dans une première étude, nous avons essayé de déterminer dans quelle mesure la présence ou l'absence de calcaire dans le profil dès la surface pouvait modifier la nutrition minérale de cette essence. Dans une deuxième étude, nous avons essayé de relier la production de l'épicéa à des caractéristiques simples du sol. Dans cette troisième étude, nous allons essayer de relier la production de l'épicéa, ou plus exactement des indices de production, aux caractéristiques de nutrition déterminées par analyses foliaires.

Il est bien évident que «l'explication» des variations de production que nous obtiendrons ne pourra être que partielle.

En effet, la production dépend d'une part des caractéristiques chimiques et d'autre part des caractéristiques physiques du sol. Or, seules les caractéristiques chimiques du sol se répercutent de façon importante sur la composition chimique des aiguilles. Nous avons vu dans un article précédent que les propriétés physiques du sol et, essentiellement, les possibilités d'alimentation en eau, jouaient un rôle considérable dans l'explication des variations de production. Dans ces conditions, nous ne pouvons espérer obtenir une explication complète de cette variation par les seules caractéristiques de nutrition, d'autant plus que, comme nous l'avons déjà noté dans ce même article, il existe une variation résiduelle importante due à la variabilité génétique de l'épicéa.

Nous ne décrirons pas les conditions de milieu de la région où nous avons travaillé, conditions qui ont largement été analysées précédemment (LE TACON-MILLIER et Decourt - Le Tacon, 1970).

Nous rappellerons seulement très brièvement les caractéristiques des sols (tableaux 1 et 2). Nous avons distingué deux grands ensembles de sols: les sols décarbonatés en partie ou totalement et les sols carbonatés. Le premier ensemble peut être subdivisé en sous-ensembles suivant la profondeur de décarbonatation : sols décarbonatés sur moins de $15 \mathrm{~cm}$ (rendzine brunifiée), sols décarbonatés entre 15 et $40 \mathrm{~cm}$ (sols bruns calciques et sols bruns eutrophes), sols décarbonatés entre 40 et $100 \mathrm{~cm}$ (sols bruns lessivés et sols lessivés), sols décarbonatés sur plus de $100 \mathrm{~cm}$ (sols lessivés) (tableau 2).

Le deuxième ensemble comprend les sous-ensembles suivants:

- sols riches en calcaire total et pauvres en matière organique - profil de type A (B) C (sols bruns calcaires) ;

- sols riches en calcaire total et riches en matière organique - profil de type $\mathrm{A} / \mathrm{C}$ (Rendzines humifères);

- sols peu carbonatés (moins de $10 \%$ de calcaire total) et peu riches en matière organique - profil de type A (B) C (sols bruns calcaires ou rendzines brunifiées peu humifères) ;

- sols carbonatés colluviáux à alimentation en eau optimale toute l'année (rendzines colluviales ou sols bruns calcaires colluviaux). 


\section{I. - METHODE DE TRAVAIL}

Les prélèvements foliaires ont été effectués au cours de l'hiver 1967-68. Nous avons retenu 81 microplacettes. Dans chacune de ces microplacettes, nous avons prélevé des aiguilles de l'année dans le tiers supérieur de la cime de cinq arbres de l'étage dominant.

Nous avons effectué une analyse totale des éléments suivant: N, $\mathrm{P}_{2} \mathrm{O}_{5}, \mathrm{~K}, \mathrm{Ca}, \mathrm{Mg}, \mathrm{Fe}$ et $\mathrm{Mn}$, soit sur les cinq prélèvements, soit sur un mélange de ces cinq prélèvements. Nous ne parlerons dans tout ce qui va suivre que des teneurs moyennes déterminées directement sur les mélanges ou déterminées à partir des cinq prélèvements analysés séparément.

Les mesures des caractéristiques dendrométriques ont été décrites dans un article précédent (DecourT-Le TaCON).

L'interprétation des résultats a été effectuée soit à l'aide de techniques statistiques simples (analyse de la variance, comparaison de moyennes) soit à l'aide de méthodes d'analyses multivariantes largement décrites par divers auteurs, par exemple TOMAssone R. pour l'analyse par régression multiple, Escouffier Y., TOMAssone R., etc... pour l'analyse des composantes principales et l'analyse par régression orthogonale.

\section{II. - RESULTATS}

Nous avions précédemment montré que la production de l'épicéa différait significativement d'un type de sol à l'autre. Un procédé très simple, pour avoir une première idée de l'influence de la nutrition sur la production, est de rechercher dans quelle mesure la nutrition diffère suivant le type de sol. I1 suffit d'effectuer une analyse de la variance à un facteur contrôlé puis une comparaison de moyennes deux à deux lorsque les différences sont globalement significatives.

Avec un tel procédé nous n'utilisons qu'une partie de l'information. Pour obtenir une meilleure explication, il est nécessaire d'utiliser l'analyse multivariate, dont les résultats feront l'objet de la deuxième partie de ce chapitre.

\section{1. - Analyse de la variance à un facteur contrôlé}

Les résultats de cette analyse sont consignés dans les tableaux 1 et 2 . Nous avons, en effet, séparé le groupe des sols carbonatés de celui des sols décarbonatés.

Dans le cas des peuplements situés sur sols carbonatés, trois éléments présentent des différences significatives suivant le type de sol. Ce sont l'azote, le potassium et le manganèse. Les peuplements situés sur les sols de type 11 et qui ont la plus basse production, sont de très loin les plus pauvres en azote. On remarquera l'amélioration considérable de la nutrition azotée sur les sols de type 12, riches en calcaire et riches en matière organique (rendzines), par rapport aux sols de type 11, riches en calcaire, mais pauvres en matière organique (sols bruns calcaires).

Dans le cas des peuplements situés sur sols décarbonatés, quatre éléments présentent des différences significatives ; ce sont le manganèse, le phosphore, le magnésium et le fer. Le manganèse et le phosphore augmentent quand la production augmente, par contre, le magnésium varie en sens inverse.

Nous noterons que les différences sont toujours significatives, deux à deux pour le manganèse, alors que pour le phosphore seuls les peuplements situés sur sols décar- 
TABLEAU 1 - TABLE 1

Sols carbonatés. Analyse de la variance et comparaison des moyennes sur les caractéristiques de nutrition en fonction des sous-ensembles de sols (Les sous-ensembles de sols qui ne présentent pas des moyennes significativement différentes au risque de $5 \%$ sont reliés par un trait) Analysis of variance on nutrition characteristics in relation to soil subclasses (carbonated soils).

(Soils subclasses, the means of which are not different at $5 \%$ level of P. are linked by a stroke)

\begin{tabular}{|c|c|c|c|c|c|c|c|c|c|}
\hline \multirow{2}{*}{$\begin{array}{l}\text { Sous- } \\
\text { groupes } \\
\text { de sols }\end{array}$} & \multirow{2}{*}{ Caractéristiques } & \multirow{2}{*}{$\begin{array}{l}\text { Production } \\
\text { moyenne à } \\
50 \text { ans } \\
\text { en } \\
\mathrm{m} 3 / \mathrm{ha} / \mathrm{an}\end{array}$} & \multicolumn{7}{|c|}{$\begin{array}{c}\text { Moyenne des teneurs en éléments minéraux } \\
\text { des aiguilles }\end{array}$} \\
\hline & & & $\mathrm{N} \%$ & $\mathrm{P}_{2} \mathrm{O}_{5} \%$ & $\mathrm{~K} \%$ & $\mathrm{Ca} \%$ & $\mathrm{Mg} \%$ & $\mathrm{Mn} \%$ & $\mathrm{Fe} \%$ \\
\hline 11 & $\begin{array}{l}\text { Calcaire total } \mathrm{A}_{1}>10 \% \text { (moyen- } \\
\text { ne } 30 \% \text { ). } \\
\text { Matière organique } \mathrm{A}_{1}<14 \% \\
\quad \text { (moyenne } 9 \% \text { ). } \\
\text { Epaisseur } \mathrm{A}_{1}<15 \mathrm{~cm} \quad \text { (moyenne } \\
\quad 11 \mathrm{~cm} \text { ). } \\
\text { Eau utile }>100 \mathrm{~mm} \quad \text { (moyenne } \\
150 \mathrm{~mm} \text { ). }\end{array}$ & 3,60 & 0,99 & $0,34-$ & $\mid-0,67$ & $0,95-1$ & $0,09-$ & $0,06-$ & $0,11-$ \\
\hline 12 & $\begin{array}{l}\text { Calcaire total } \mathrm{A}_{1}>10 \% \text { moyen- } \\
\text { ne } 26 \% \text { ). } \\
\text { Matière organique } \mathrm{A}_{1}>14 \% \\
\quad(\text { moyenne } 18 \% \text { ). } \\
\text { Epaisseur } \mathrm{A}_{1}>15 \mathrm{~cm} \quad \text { (moyenne } \\
36 \mathrm{~cm} \text { ). } \\
\text { Eau utile faible (moyenne } 90 \mathrm{~mm} \text { ). }\end{array}$ & $6,40-$ & $1,307-$ & $0,34-$ & 0,57 & $0,83 \ldots$ & $0,105-$ & $0,06-1$ & $0,12-$ \\
\hline 13 & $\begin{array}{l}\text { Calcaire total }<10 \% \text { (moyenne } \\
3 \% \text { ). } \\
\text { Matière organique } \mathrm{A}_{1}: \text { moyenne } \\
15 \% \text {. } \\
\text { Epaisseur } \mathrm{A}_{1}: \text { moyenne } 15 \mathrm{~cm} \text {. } \\
\text { Eau utile comprise entre } 50 \text { et } \\
150 \mathrm{~mm} \text {. }\end{array}$ & 6,80 & 1,357 & 0,34 & $|-0,70-|$ & $0,87-$ & $0,101-$ & $0,10-$ & $0,13-$ \\
\hline 14 & $\begin{array}{l}\text { Teneur en calcaire total: moyen- } \\
\text { ne } 16 \% \text {. } \\
\text { Eau utile: moyenne } 160 \mathrm{~mm} \text {. } \\
\text { Alimentation en eau optimale } \\
\text { toute l'année en raison d'ap- } \\
\text { ports latéraux. }\end{array}$ & 11,90 & $1,370_{-}$ & $0,37-$ & $0,68-$ & $0,81 \ldots$ & 0,09 & $0,07 \ldots$ & $0,08-$ \\
\hline $\begin{array}{c}\text { Test } \\
\text { F }\end{array}$ & & $* *$ & $*$ & & $*$ & & & $\%$ & \\
\hline
\end{tabular}




\section{TABLEAU 2 - TABLE 2}

Sols décarbonatés. Analyse de la variance et comparaison des moyennes sur les caractéristiques de

(Les sous-ensembles de sols qui ne présentent pas des moyennes significativement différentes au

risque de $5 \%$ sont reliés par un trait)

Analysis of variance on nutrition characteristics in relation to soil subclasses (decarbonated soils)

(Soils subclasses, the means of which are not different at $5 \%$ level of P. are linked by a stroke)

\begin{tabular}{|c|c|c|c|c|c|c|c|c|c|}
\hline \multirow{2}{*}{$\begin{array}{l}\text { Groupes } \\
\text { de sol }\end{array}$} & \multirow{2}{*}{ Caractéristiques } & \multirow{2}{*}{$\begin{array}{l}\text { Moyenne } \\
\text { des produc- } \\
\text { tions à } \\
50 \text { ans } \\
\text { en } \\
\mathrm{m}^{3} / \mathrm{ha} / \mathrm{an}\end{array}$} & \multicolumn{7}{|c|}{$\begin{array}{c}\text { Moyenne des teneurs en éléments minéraux } \\
\text { des aiguilles }\end{array}$} \\
\hline & & & $\mathrm{N} \%$ & $\mathrm{P}_{2} \mathrm{O}_{5} \%$ & $\mathrm{~K} \%$ & $\mathrm{Ca} \%$ & $\mathrm{Mg} \%$ & Mn $/ \%$ & $\mathrm{Fe} / \%$ \\
\hline 22 & $\begin{array}{c}\text { So s décarbonatés } \\
15 \text { et } 40 \mathrm{~cm}\end{array}$ & 11,00 & 1,44 & $0,33-$ & $0,69-$ & $0,81-$ & 0,12 & 0,43 & 0,11 \\
\hline 23 & $\begin{array}{l}\text { Sols décarbonatés } \\
\text { entre } 40 \text { et } 100 \mathrm{~cm}\end{array}$ & 14,40 & $1,40-$ & 0,37 & $0,67-$ & 0,74 & 0,09 & 0,59 & $0,16-$ \\
\hline 24 & $\begin{array}{l}\text { So's décarbonatés } \\
\text { sur plus de } 100 \mathrm{~cm}\end{array}$ & 16,00 & $1,48-$ & 0,39 & $0,67-$ & $0,71-$ & $0,08 \ldots$ & 0,66 & $0,14 \ldots$ \\
\hline Test F & & $* *$ & & $*$ & & & $*$ & $* *$ & $*$ \\
\hline
\end{tabular}


bonatés de 15 à $40 \mathrm{~cm}$ et les peuplements situés sur sols entièrement décarbonatés, présentent des différences significatives. Pour le magnésium, il n'existe pas de différence significative entre les peuplements situés sur les types de sols 23 et 24 .

\section{2. - Analyses multivariates}

Par l'analyse de la variance, nous n'avons utilisé que les moyennes des teneurs en éléments minéraux des aiguilles par type de sol. Par analyses multivariates, nous allons pouvoir utiliser une plus grande partie de l'information, mais nous allons perdre la facilité d'interprétation fournie par le «découpage» en type de station.

\subsection{Ensemble des peuplements.}

2.211. Liaisons entre caractéristiques des peuplements nutrition et âge. Nous avons effectué une analyse par régression progressive sur trois variables à expli-

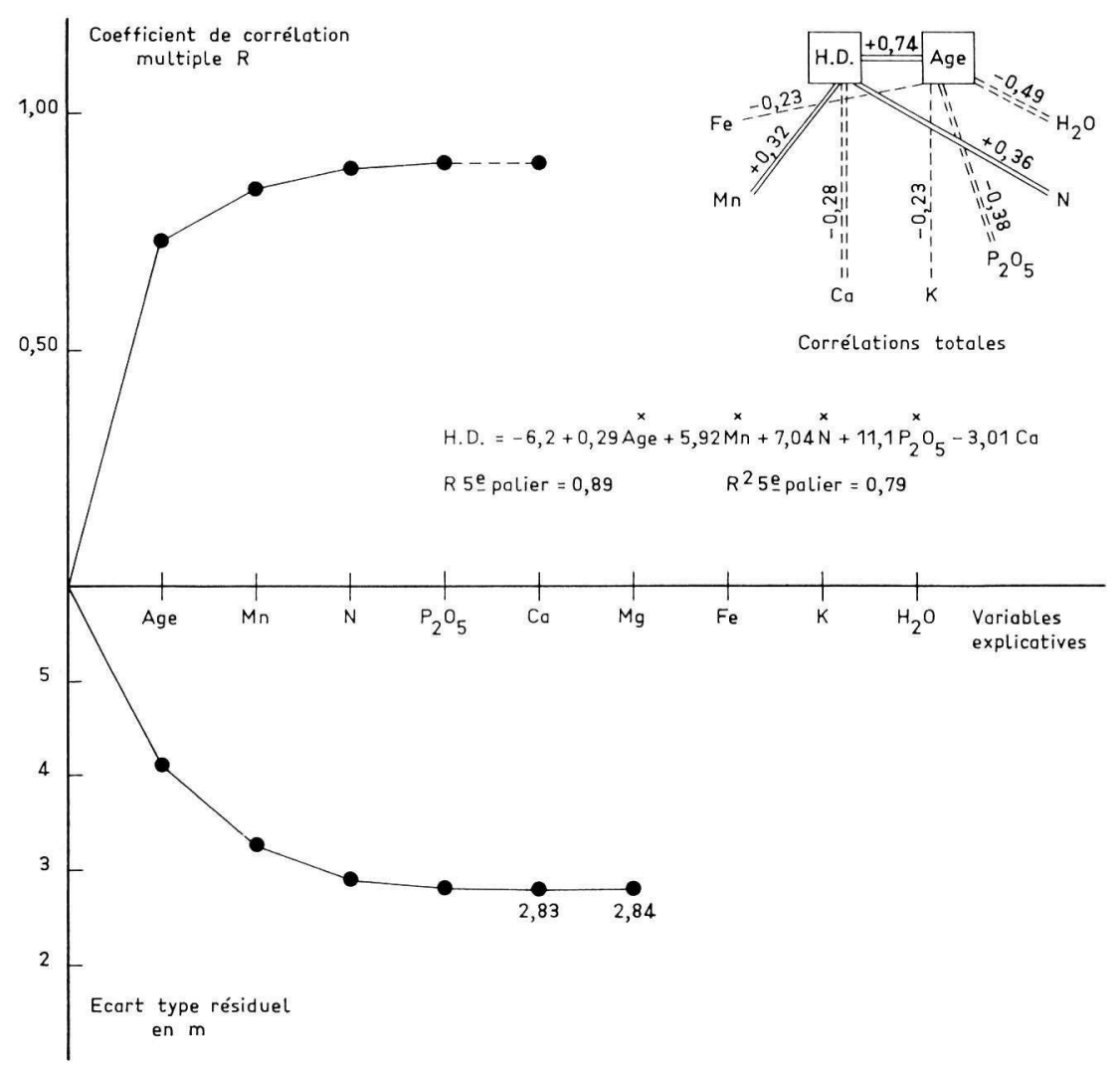

FIG. 1. - Régression progressive entre hauteur dominante, caractéristiques de nutrition et âge (ensemble des peuplements)

Fig. 1. - Multiple progressive regression between dominant height, nutrition and age (81 stands) 
quer : hauteur dominante, circonférence dominante et circonférence moyenne. Les équations obtenues pour ces trois variables sont très semblables, mais le pourcentage d'explication le meilleur a été obtenu comme nous pouvions nous y attendre avec la hauteur dominante. Nous ne donnerons donc que les résultats concernant la hauteur dominante (figure 1).

La hauteur dominante est évidemment très liée à l'âge $(r=0,74)$. Elle est également liée positivement à l'azote et au manganèse et négativement au calcium. On remarquera que l'âge est lié négativement à la teneur en eau, phosphore, potassium et fer (corrélations totales).

Par régression progressive, nous estimons la hauteur dominante en introduisant successivement les variables explicatives une à une. Si nous arrêtons la régression lorsque l'écart-type résiduel devient minimal, nous obtenons une équation à 5 variables, comprenant dans l'ordre, l'âge, le manganèse, l'azote, le phosphore et le calcium, ce dernier élément n'intervenant pas de manière significative dans la régression. Par contre, le phosphore intervient de manière significative alors qu'il n'existe pas de corrélation totale significative entre production et phosphore.

Au cinquième palier, le coefficient de corrélation multiple est de 0,89 , ce qui correspond à une «explication» de la production de l'ordre de $80 \%$.

En fait, seule la variation de hauteur dominante non expliquée par l'âge nous intéresse ici; pour cette raison, nous avons voulu nous «affranchir» de l'âge en travaillant sur des variables peu dépendantes de l'âge ou ramenées à âge constant.

2.212. Liaisons entre les caractéristiques des peuplements ramenées à âge constant et la nutrition. - Nous avons pris comme variables à expliquer la hauteur dominante à 20 ans, la hauteur dominante à 35 ans, la hauteur dominante à 50 ans et une estimation de la vitesse de croissance obtenue par la tangente de l'angle formé par la partie rectiligne des courbes de croissance et l'axe des $x$ (temps).

Toutes ces variables ont été déterminées par analyses de tige.

Nous avons obtenu respectivement par régression progressive pour chacune de ces variables un coefficient de corrélation multiple de 0,67 (Hd 20 ans), 0,758 (Hd 35 ans), 0,772 ( $\mathrm{Hd} 50$ ans) et 0,662 (tangente). Nous ne gardons ici que la meilleure estimation, c'esî-à-dire celle obtenue pour la hauteur dominante à 50 ans qui nous donne un pourcentage d'explication de $60 \%$ environ.

Il reste $40 \%$ de la variation de hauteur dominante non explicable par les caractéristiques de nutrition. Dans ces $40 \%$, entrent l'influence des éléments que nous n'avons pas dosés, les erreurs d'échantillonnage, les erreurs analytiques et les erreurs de mesures, les propriétés physiques des sols, l'évolution des différents caractères en fonction de l'âge, la variation génétique et les erreurs de modèle.

Il existe un coefficient de corrélation totale très significatif et positif entre $\mathrm{Hd}$ 50 ans et dans l'ordre $\mathrm{N}(0,60)$, $\mathrm{Mn}(0,59)$ et $\mathrm{P}_{2} \mathrm{O}_{5}(0,39)$. Par contre avec le calcium, le coefficient de corrélation est négatif et très significatif $(-0,42)$.

Par régression progressive, quand l'écart-type résiduel est minimal, nous obtenons une équation à 5 variables $\left(\mathrm{N}, \mathrm{Mn}, \mathrm{P}_{2} \mathrm{O}_{5}, \mathrm{Ca}\right.$ et $\left.\mathrm{Mg}\right)$ où seuls interviennent significativement $\mathrm{N}, \mathrm{Mn}$ et $\mathrm{P}_{2} \mathrm{O}_{5}$ (figure 2 et tableau 4). 


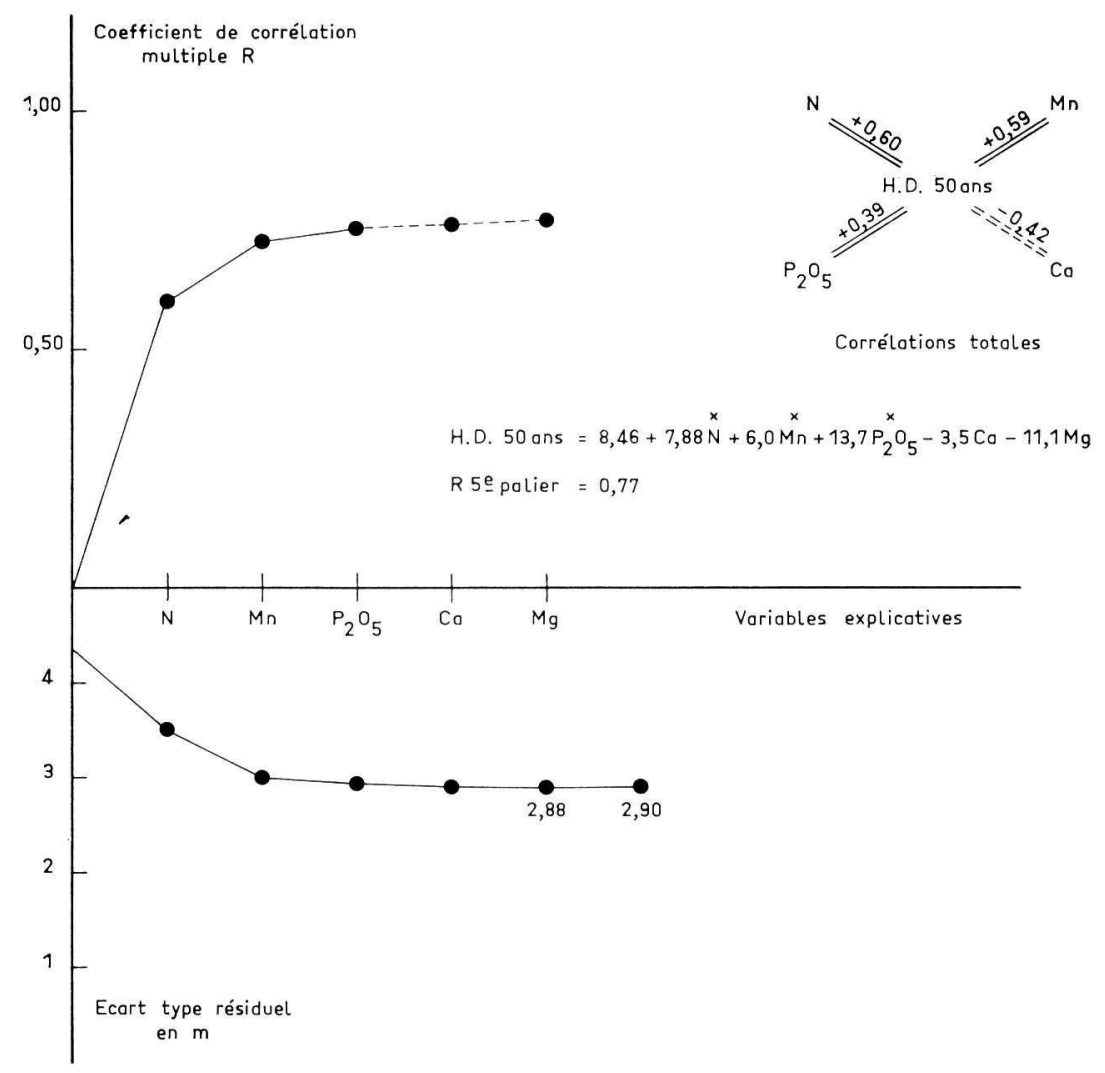

FIG. 2. - Régression progressive entre hauteur dominante à 50 ans et caractéristiques de nutrition (ensemble des peuplements)

FIG. 2. - Multiple progressive regression between dominant height at age 50 and mineral nutrition (stands situated on carbonated soils)

\section{- Régression orthogonale.}

Nous avons ensuite effectué une nouvelle régression dite orthogonale de la hauteur dominante à 50 ans en fonction des composantes principales qui sont, rappelons-le, des combinaisons non corrélées des variables de départ. Les résultats de la régression orthogonale sont consignés dans le tableau 3.

Nous n'avons conservé que les quatre composantes ayant les valeurs propres les plus élevées. Sur ces quatre composantes, deux sont très significativement liées à la hauteur dominante et une légèrement liée à cette même hauteur dominante.

La première composante représente le phénomène de fond associant positivement $\mathrm{N}, \mathrm{P}_{2} \mathrm{O}_{5}, \mathrm{Mn}$ et négativement $\mathrm{Ca}$. Elle contribue très largement à expliquer la production $(\mathrm{t}=8,59 * *)$.

La deuxième composante caractérise la liaison particulière $\mathrm{Mn}-\mathrm{Fe}$ à laquelle participe négativement $\mathrm{K}$. Cette composante est légèrement liée à la production. On peut considérer que l'analyse des composantes principales dissocie l'effet du man- 


\section{TABLEAU 3 - TABLE 3}

Analyse par régression orthogonale. Ensemble des peuplements. (Variable expliquée: HD 50. Variables explicatives: caractéristiques de nutrition)

Explication of stand dominant height at age 50 and mineral composition of needles by orthogonal regression ( 81 stands)

\begin{tabular}{c|c|c|l|c|c}
\hline $\begin{array}{c}\text { Compo- } \\
\text { sante }\end{array}$ & $\begin{array}{c}\text { Valeur } \\
\text { propre }\end{array}$ & $\begin{array}{c}\text { Pourcen- } \\
\text { tage } \\
\text { cumulé }\end{array}$ & $\begin{array}{c}\text { Variables initiales intervenant } \\
\text { dans la composante }\end{array}$ & $\begin{array}{c}\text { Test de } \\
\text { Student }\end{array}$ & $\begin{array}{c}\text { Coefficient } \\
\text { de } \\
\text { régression }\end{array}$ \\
\cline { 3 - 4 } & 2,23 & 27,9 & $+P_{2} O_{5},+M n,+N,-\mathrm{Ca}$ & $8,59 * *$ & 0,66 \\
2 & 1,46 & 46,1 & $-K,+F e,+\mathrm{Mn}$ & $2,80 *$ & 0,21 \\
3 & 1,21 & 61,3 & $+\mathrm{Ca},+\mathrm{Mg}$ & $-3,13 * *$ & 0,24 \\
4 & 0,87 & 72,2 & $+M g$ & 1,15 & 0,09 \\
\hline \hline
\end{tabular}

ganèse en deux parties. La première partie correspond à la liaison avec le calcium (antagonisme calcium-manganèse), la seconde à la liaison avec le fer ( $2^{\mathrm{e}}$ composante).

L'introduction de $\mathrm{Mn}$ au deuxième palier de la régression progressive caractérise à la fois l'effet de la deuxième composante principale, c'est-à-dire la part de Mn lié au fer et l'effet de la liaison de Mn avec le calcium restant après le premier palier.

La troisième composante fait intervenir le calcium et le magnésium ensemble. Comme l'indique la quatrième composante, le magnésium n'a aucun effet sur la production. On peut donc avec précaution, considérer la troisième composante comme représentant l'apport spécifique du calcium.

Dans cette analyse par régression orthogonale, il y a donc eu dissociation de l'effet propre du calcium qui était en partie masqué dans l'analyse par régression progressive par ses effets secondaires (nutrition en $\mathrm{Mn}$ et $\mathrm{N}$ ).

La régression progressive n'avait pu mettre en évidence ce rôle propre du calcium qui est ainsi parfaitement mis en lumière par régression orthogonale.

\section{TABLEAU 4 - TABLE 4}

Analyse par régression multiple progressive. Ensemble des peuplements. (Variable expliquée: HD 50. Variables explicatives: caractéristiques de nutrition)

Explication of stand dominant height at age 50 in relation to mineral composition of needles by multiple progressive regression (81 stands)

Palier $\mathrm{n}^{\circ} 5$

Coefficient de corrélation multiple: 0,770

Terme constant : 8,458

\begin{tabular}{|c|c|c|}
\hline Variable & Coefficient de régression & Test de Student \\
\hline Azote $\ldots \ldots \ldots \ldots \ldots \ldots$ & $+7,88$ & $+4,46 * *$ \\
\hline Phosphore $\quad \ldots \ldots \ldots \ldots \ldots$ & $+13,72$ & $+2,65 *$ \\
\hline Calcium $\quad \ldots \ldots \ldots \ldots \ldots$ & $-3,48$ & $-1,49$ \\
\hline Magnésium $\ldots \ldots \ldots \ldots \ldots$ & $-11,14$ & $-1,29$ \\
\hline Manganèse $\ldots \ldots \ldots \ldots \ldots$ & $+6,00$ & $+4,63 * *$ \\
\hline
\end{tabular}




\subsection{Dissociation en fonction des types de sols.}

Il nous est apparu intéressant d'essayer de dissocier l'action des caractéristiques de nutrition sur la croissance en fonction des types de sols. Il est, en effet, évident que les divers éléments ont des rôles très différents suivant les conditions de sols. Nous avons donc effectué pour chaque ensemble et sous-ensemble de sols une analyse par régression progressive. Pour les deux grands ensembles (sols carbonatés et sols décarbonatés), l'analyse est encore valable, car nous disposons à chaque fois d'une quarantaine d'échantillons. Par contre, lorsque nous passons aux sous-ensembles, nous ne disposons plus que d'une dizaine d'échantillons, ce qui est très insuffisant et met en cause la validité des régressions.

2.221. Nutrition et hauteur dominante à l'intérieur des peuplements situés sur sols carbonatés. - Deux éléments, $\mathrm{N}$ et $\mathrm{P}: \mathrm{O}_{2 .,}$, ont un coefficient de corrélation totale positif et très significatif avec la hauteur dominante alors que le calcium présente un coefficient de corrélation total significatif et négatif. Dans la régression progressive, seule l'introduction de $\mathrm{N}$ et $\mathrm{P}_{2} \mathrm{O}_{\text {; }}$ est significative. Le coefficient de corrélation multiple est de 0,71 au quatrième palier, lorsque l'écart-type résiduel est minimal. Le pourcentage d'explication devient faible (50\% environ). Nous avons comparé les valeurs mesurées et les valeurs calculées à l'aide de l'équation de régression multiple. Nous constatons que tous les peuplements situés sur sols à forte alimentation en eau sont très sous-estimés par le modèle. La raison en est fort simple, le supplément de production est obtenu grâce aux suppléments d'eau, ce qui ne se répercute absolument pas sur la nutrition. Il en résulte une réduction du pourcentage d'explication de la production par ces seules variables (figure 3 ).

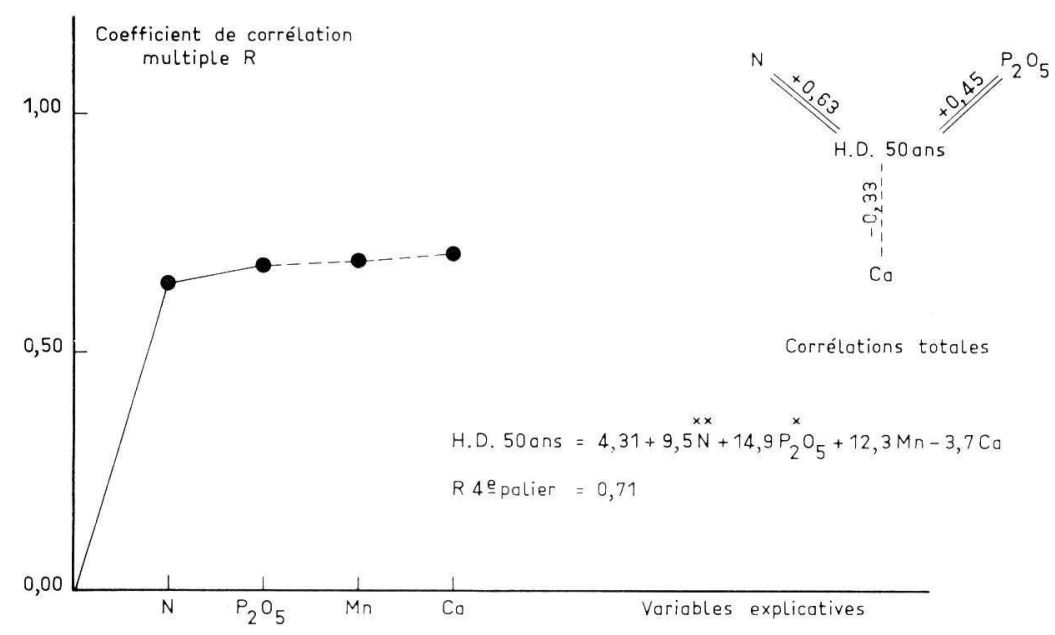

FIG. 3. - Régression progressive entre hauteur dominante à 50 ans et caractéristiques de nutrition (Peuplements situés sur sols carbonatés)

FIG. 3. - Multiple progressive regression between dominant height at age 50, and mineral nutrition. (Stands situated on carbonated soils) 
2.222. Nutrition et hauteur dominante à l'intérieur des peuplements situés sur sols décarbonatés. - Seuls, le fer et le manganèse présentent des coefficients de corrélation totaux significatifs avec la hauteur à 50 ans.

Dans la régression progressive, le fer qui est très lié au manganèse disparaît. Par contre, l'introduction du magnésium devient significative après l'introduction du manganèse. L'introduction de ces deux éléments $\mathrm{Mn}$ et $\mathrm{Mg}$ est seule significative (figure 4).

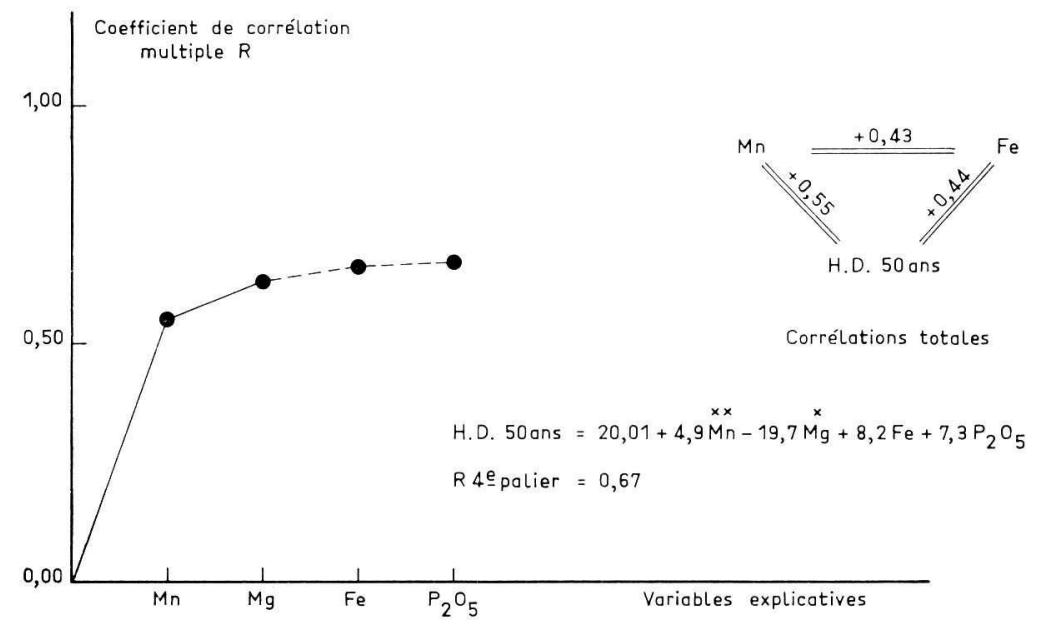

FIG. 4. - Régression progressive entre hauteur dominante à 50 ans et caractéristiques de nutrition (Peuplements situés sur sols décarbonatés)

FIG. 4. - Multiple progressive regression between dominant height at age 50 and mineral nutrition (stands situated on decarbonated soils)

\section{TABLEAU 5 - TABLE 5}

Coefficients de corrélation totaux entre hauteur dominante à 50 ans et caractéristiques de nutrition à l'intérieur des sous-ensembles de sols

Total correiation coefficients between stand dominant height at age 50 and mineral composition of needles in each soil subclass

\begin{tabular}{|c|c|c|c|c|c|c|c|}
\hline \multirow{2}{*}{$\begin{array}{l}\text { Sous- } \\
\text { ensembles } \\
\text { de sols }\end{array}$} & \multicolumn{7}{|c|}{ Coefficients de corrélation totaux avec HD 50 ans } \\
\hline & $\mathrm{N}$ & $\mathrm{P}_{2} \mathrm{O}_{5}$ & $\mathrm{~K}$ & $\mathrm{Ca}$ & $\mathrm{Mg}$ & $\mathrm{Mn}$ & $\mathrm{Fe}$ \\
\hline 11 & $* *$ & $*$ & NS & NS & $*$ & NS & NS \\
\hline 12 & NS & NS & NS & NS & NS & NS & NS \\
\hline 13 & NS & $*$ & NS & NS & NS & NS & NS \\
\hline 14 & NS & NS & NS & NS & $*$ & NS & NS \\
\hline 22 & NS & NS & NS & NS & NS & NS & NS \\
\hline 23 & NS & NS & NS & NS & NS & NS & NS \\
\hline 24 & NS & $*$ & NS & NS & NS & NS & NS \\
\hline
\end{tabular}


Le pourcentage d'explication est également faible, il est en effet de $45 \%$ environ au quatrièmé palier. Nous reviendrons sur cet aspect du problème dans la discussion.

2.223. Nutrition et hauteur dominante à l'intérieur des différents types de sol. - Nous avons indiqué précédemment que ces calculs sont fortement sujets à caution en raison du trop petit nombre d'observations par type de sol.

Nous nous contenterons de donner un tableau des coefficients de corrélation totaux significatifs entre hauteur dominante et nutrition pour chaque type de sol (tableau 5).

\section{DISCUSSION}

Il semble bien que le niveau de nutrition en azote soit le plus lié avec la production.

Nous avions déjà vu que le niveau de nutrition en azote était plus élevé sur sols décarbonatés que sur sols carbonatés. Or, la production est nettement supérieure sur les sols décarbonatés. Nous pouvons donc penser que cette diminution de production provoquée par la présence de calcaire dès la surface s'explique en partie par une diminution de la disponibilité en azote.

Si une meilleure production est observée sur sol décarbonaté et si ce gain de production peut être attribué en partie à une amélioration de la nutrition azotée, à l'intérieur des peuplements situés sur sols décarbonatés, les variations de teneur en azote des aiguilles sont faibles et ne contribuent pas à expliquer les variations de production.

Par contre, les variations de production existant sur les sols carbonatés apparaissent très liées au niveau de nutrition azotée. $40 \%$ environ de la variation de production sont expliqués uniquement par le niveau de nutrition azotée, ce qui est considérable. En effet, pour une partie de ces sols (sols colluviaux), l'amélioration de la production est due uniquement aux apports d'eau supplémentaires.

Le niveau de nutrition en azote des peuplements situés sur sols de type 12 (sols riches en calcaire et en matière organique) est nettement supérieur à celui des peuplements situés sur les sols de type 11 (sols riches en calcaire et pauvres en matière organique). Nous pouvons donc penser que la matière organique qui compense l'action néfaste du calcaire agit en améliorant la nutrition azotée. Rappelons que dans un article précédent (DeCourt, LE TACON), nous avions supposé que la matière organique compensait l'action du calcaire en isolant le système radiculaire des particules de carbonate de calcium et en neutralisant par ses groupements acides, une partie des carbonates. Un troisième mode d'action possible apparaît donc ainsi.

Après l'azote, l'élément qui semble intervenir le plus dans l'explication des variations de production est le manganèse. Pour l'ensemble des peuplements, son coefficient de corrélation totale avec la hauteur dominante à 50 ans est pratiquement aussi élevé que celui de l'azote $(0,59$ contre 0,60$)$.

Le niveau de nutrition en manganèse est systématiquement faible en sol carbonaté et descend même assez fréquemment au-dessous du seuil de carence dans les peupléments situés sur les sols les plus riches en calcaire. Nous pouvons donc penser que les difficultés de croissance de l'épicéa sur les sols les plus carbonatés proviennent en partie des difficultés d'alimentation en manganèse. La liaison production nutrition en 
manganèse, qui est mise en évidence par les différents calculs a donc globalement une signification réelle. Mais si nous examinons les résultats des analyses statistiques effectuées sur chacun des deux ensembles de peuplements, nous allons voir que cette signification doit être considérée avec beaucoup de prudence.

Les calculs effectués sur la première population (peuplements à sols carbonatés) ne font apparaître aucune influence du manganèse sur la production (coefficient de corrélation totale non significatif et introduction dans la régression progressive multiple non significative). L'explication en est assez claire. En raison de la rétrogradation du manganèse dans le sol et du bloquage de son absorption par le calcium, le niveau de nutrition est toujours très bas et peu variable; il ne peut donc être lié statistiquement à la production, bien que par sa déficience au moins dans les sols les plus carbonatés, il contribue partiellement à maintenir la production à un niveau très bas.

Au contraire, les calculs effectués sur la seconde population (peuplements à sols décarbonatés) font apparaître une liaison très significative entre production et nutrition en manganèse (coefficient de corrélation totale très significatif et introduction dans la. régression progressive multiple très significative). Mais, s'il y a liaison statistique, il ne doit y avoir en réalité aucune liaison de cause à effet à l'intérieur de cette population entre nutrition en manganèse et production. En effet, dès que le sol est décarbonaté, le niveau de manganèse dans les aiguilles devient suffisant. Quand la profondeur de décarbonatation augmente, le sol se désature et la disponibilité en calcium échangeable diminue. En raison de l'antagonisme calcium-manganèse, la décarbonatation se traduit donc par une augmenttion de l'absorption du manganèse par l'épicéa.

Mais cette augmentation correspond à une «consommaiton de luxe » et ne doit avoir aucun effet sur la croissance.

Par contre, quand la profondeur de décarbonatation et, corrélativement, la profondeur totale du sol augmentent, la disponibilité en eau du sol augmente, ce qui doit se traduire par une augmentation de la production.

Production et niveau de nutrition en manganèse varient donc dans le même sens parce qu'ils sont dépendants d'un même facteur, c'est-à-dire, la profondeur de décarbonatation; mais, s'il y a liaison statistique, il n'y a pas obligatoirement liaison de causalité.

Après l'azote et le manganèse, un troisième élément, le phosphore, apparaît lié à la production de l'épicéa, mais de façon beaucoup moins étroite. Pour l'ensemble des peuplements, le coefficient de corrélation totale entre niveau de nutrition en phosphore et: hauteur dominante à 50 ans est de 0,39 , ce qui est néanmoins très significatif. Dans la régression progressive, l'introduction du phosphore se fait au troisième palier et est significative.

Quand nous disjoignons les deux types de peuplements, il apparaît que le phosphore joue un rôle très différent suivant que nous sommes en sol carbonaté ou en sol décarbonaté.

Dans les peuplements situés sur sols carbonatés, le coefficient de corrélation totale entre hauteur dominante et $\mathrm{P}_{2} \mathrm{O}_{5}$ des aiguilles est de 0,45 et dans la régression multiple. le phosphore est, avec l'azote, le seul élément qui soit significatif. Le phosphore joue donc un rôle très important dans l'explication des variations de production en sol carbonaté. Nous pouvons admettre qu'une disponibilité élevée du sol en phosphore 
en sol carbonaté permet d'améliorer la production et de contrecarrer en partie l'action du calcaire. En examinant une à une les différentes micro-placettes situées sur sols carbonatés, nous avons pu constater que certains peuplements avaient, compte tenu de la teneur en calcaire du sol, une production élevée ce qui correspondait effectivement à une teneur élevée en phosphore dans le sol et dans les aiguilles.

Au contraire, dans les peuplements situés sur sols décarbonatés en partie, le phosphore ne semble plus guère jouer de rôle dans l'explication des variations de production au moins si nous considérons tous les peuplements situés sur sols décarbonatés totalement ou en partie. En effet, pour ces sols, il est très probable que ce sont les propriétés physiques, en particulier la disponibilité en eau, qui est liée à la profondeur de décarbonatation, qui expliquent en premier lieu les variations de production, d'autant plus que le niveau de nutrition en azote et manganèse est dans l'ensemble assez constant. Par contre, dans le cas des sols entièrement décarbonatés, donc très profonds, et où la variation de teneur en phosphore du sol est très importante, le phosphore des aiguilles apparaît très lié à la production.

Les trois éléments qui apparaissent le plus liés avec la production, que ces liaisons soient ou non des liaisons de cause à effet, sont donc l'azote, le manganèse et le phosphore.

Il est remarquable de constater que dans l'analyse par régression orthogonale, que nous avons effectuée sur l'ensemble des peuplements, ces trois éléments surtout interviennent dans la première composante qui est la plus liée avec la hauteur dominante (coefficient de corrélation de 0,66 et test de Student de $+8,59$ ). L'analyse par rágression orthogonale confirme donc les analyses précédentes, mais de plus, met mieux en lumière le rôle du calcium.

Apparemment, le niveau de nutrition en calcium ne paraît pas très lié avec la production. Pour l'ensemble des peuplements, s'il existe un coefficient de corrélation total négatif et significatif, dans le cas de la hauteur dominante non ramenée à âge constant, ce coefficient n'est plus significatif dans le cas de la hauteur dominante a 50 ans. D'autre part, dans les deux régressions multiples progressives effectuées sur la hauteur dominante et la hauteur dominante à 50 ans, l'introduction du calcium n'est jamais significative.

Ce fait provient très probablement du faible pouvoir discriminant du calcium, dont l'absorption est considérablement freinée lorsque sa disponibilité dans le milieu augmente (LE TACON-Millier, 1970). D'autre part, lonsque la disponibilité en calcium du milieu augmente, il y a diminution du niveau de nutrition en manganèse et en azote des aiguilles (liaisons négatives $\mathrm{Ca}-\mathrm{Mn}$ et $\mathrm{Ca}-\mathrm{N}$ ). Dans l'analyse par régression progressive ce sont l'azote et le manganèse qui sont introduits en premier. Leur introduction absorbe une grande partie de la variation propre du calcium dont le rôle ne peut plus ainsi apparaître.

L'analyse des composantes principales a permis d'isoler la variation due à $\mathrm{N}$ et et à Mn dans la première composante et la variation propre du calcium ou au moins une partie dans la troisième composante.

Dans la régression orthogonale, la troisième composante est liée significativement à la hauteur dominante à 50 ans ce qui permet de mettre en évidence l'effet propre du calcium qui n'apparaissait pas dans la régression progressive. 
L'azote, le calcium, le phosphore et le manganèse sont donc les quatre éléments essentiels intervenant dans l'explication des variations de production.

Les trois autres éléments n'apparaissent pas jouer de rôle important. Le potassium est toujours à un niveau satisfaisant, sauf peut-être dans les peuplements situés sur rendzines et n'apparaît jamais intervonir de manière certaine.

On notera cependant que le potassium intervient négativement dans la deuxième composante principale et semblerait donc présenter avec la production, une légère liaison négative. Cette liaison n'est cependant jamais significative lorsque l'on fait intervenir le potassium seul (coefficient de corrélation totale ou partielle entre hauteur dominante et potassium jamais significatif).

Le rôle du fer, s'il n'apparaît pas comme particulièrement important, pourrait peut-être être moins négligeable que celui du potassium. Le fer en effet interviènt positivement dans la deuxième composante principale qui caractérise la liaison particulière $\mathrm{Mn}-\mathrm{Fe}$ et qui est légèrement liée positivement à la production.

Le magnésium ne paraît pas non plus intervenir. Nous noterons cependant que dans la régression progressive effectuée sur les peuplements situés sur sols décarbonatés, bien qu'il n'existe pas de coefficient de corrélation total significatif entre $\mathrm{Hd}$ 50 ans et teneur en magnésium des aiguilles, l'introduction du magnésium est légèrement significative. Mais nous ne pensons pas, dans ce type de sol, qu'il y ait une quelconque liaison de cause à effet entre nutrition en magnésium et production.

Il faut souligner l'absence de liaison ou les liaisons très faibles existant entre production et niveau de nutrition en potassium, magnésium et fer sur ce type de substrat riche en calcium et parfois carbonaté. D’une manière générale, chez les végétaux, on a souvent insisté en sol calcaire sur les difficultés de nutrition en fer, en magnésium et en potassium. La chlorose ferrique est bien connue et on a souvent parlé d'un antagonisme $\mathrm{Ca}-\mathrm{K}$ et $\mathrm{Ca}-\mathrm{Mg}$. Dans le cas de l'épicéa, un excès de calcium dans le milieu ne se traduit pas par une diminution notable du niveau de nutrition en ces trois éléments. Un certain antagonisme $\mathrm{Ca}-\mathrm{Mg}$ peut néanmoins être mis en évidence en sol carbonaté. Il existe en effet un coefficient de corrélation totale légèrement significatif entre teneur en calcium et teneur en magnésium des aiguilles sur sol carbonaté. Enfin, il faudrait peut-être associer aux difficultés de nutrition en manganèse de l'épicéa sur les sols les plus carbonatés, une certaine difficulté de nutrition en fer, mais sans que cela prenne une importance considérable. 


\section{CONCLUSION}

La croissance de l'épicéa sur les plateaux calcaires de l'est de la France est très liée aux conditions d'alimentation minérale.

L'azote apparaît comme l'élément le plus important. Nous pouvons, selon toute vraisemblance, attribuer le gain de production observé en sol décarbonaté par rapport à la production en sol carbonaté, en partie à une amélioration du niveau de nutrition azotée. Mais en sol décarbonaté la nutrition azotée reste à un niveau moyen quel que soit le sol et ne contribue pas à expliquer la variation de production observée à l'intérieur de cette population. Par contre en sol carbonaté la croissance est très liée aux possibilités d'alimentation en azote, en particulier sur rendzine il y a une nette amélioration de la teneur en azote des aiguilles par rapport à ce que l'on observe sur des sols bruns calcaires aussi riches en calcaire que les rendzines, mais nettement plus pauvres en matière organique.

La chute de production observée en sol carbonaté peut également être en partie attribuée à une déficience en manganèse qui peut être très marquée et descendre au-dessous du seuil de carence sur les sols les plus riches en calcaire. Statistiquement, les variations de production à l'intérieur des sols carbonatés ne sont pas liés à la nutrition en manganèse, alors que c'est exactement l'inverse en sol décarbonaté. En réalité, il n'y a aucune relation de causalité en sol décarbonaté entre teneur en manganèse des aiguilles et production.

Le phosphore apparaît également très important mais contribue surtout à expliquer les variations de production à l'intérieur des peuplements situés sur sols carbonatés. Un niveau de nutrition élevé en phosphore peut permettre de compenser dans une certaine mesure un excès de calcaire.

La teneur en calcium des aiguilles est liée négativement à la croissance. Cette liaison négative est assez difficile à mettre en évidence car l'effet d'une augmentation de teneur en calcium et en calcaire du milieu, ne se répercute que très faiblement sur le niveau de nutrition en calcium par freinage de l'absorption de cet élément. Par contre, cette augmentation de la disponibilité en calcium et en calcaire du milieu se répercute sur la nutrition en azote et en manganèse, ce qui masque l'action propre du calcium au niveau des aiguilles. Par régression orthogonale, il est néanmoins possible de dissocier en partie l'effet propre du calcium de ces effets secondairés, ce qui n'est pas possible par régression progressive.

Enfin, le niveau de nutrition en potassium, magnésium et fer ne contribue que très peu à expliquer les variations de production de l'épicéa. 


\section{SUMMARY}

INFLUENCE OF MINERAL NUTRITION

\section{ON GROWTH OF NORWAY SPRUCE ON CALCAREOUS TABLE-LANDS IN EASTERN FRANCE}

Growth of Norway Spruce on calcareous table-lands in Eastern France is intimately delated to mineral nutrition.

Nitrogen seems to be the most important element. The yield of Norway Spruce on decarbonated soils is greater than on carbonated soils. This may apparently be attributed partly to an improved nitrogen nutrition level. On decarbonated soils, however, nitrogen nutrition remains at a medium level, whatever the type of soil may be, and does not allow to explain variations of yield among this population.

On rendzina, nitrogen content of needles is markedly higher than the one existing on calcareous brown soils, which are equally rich in limestone but much poorer in organic matter than rendzina.

Yield decrease on carbonated soils may be also attributed partly to a manganese deficiency, since on the most calcareous soils manganese contents of needles below minimum level.

According to statistics, variations among carbonated soils are not related to manganese nutrition, whereas for decarbonated soils the situation is exactly opposite.

But in reality, no cause and effect relation could be shown between manganese content of needles and yield on decarbonated soils.

Phosphorus is very important as well, but allows above all to explain yield variation among stands on carbonated soils. To a certain extent, a high phosphorus nutrition level may compensate an excessive quantity of limestone.

Calcium content of needles is negatively related to growth. This negative relation is rather difficult to be shown since an important increase of calcium or calcium carbonate content in soil provokes no more than a small increase of calcium content needles.

This increase, however, of calcium or limestone content in soil modifies considerably nitrogen and manganese nutrition, which increase maskes the specific action of calcium in needles.

By othogonal regression it is, however, possible to dissociate partly the specific action of calcium from its secondary actions, which dissociation is not possible by p:ogressive regression.

Potassium, magnesium and iron nutrition levels contribute very little to explain yield variations.

\section{ZUSAMMENFASSUNG}

DER EINFLUSS DER MINERALSTOFFERNÄHRUNG AUF DAS WACHSTUM DER FICHTE AUF DEN KALKPLATEAUS IN OSTFRANKREICH

Das Wachstum der Fichte auf den Kalkplateaus in Ostfrankreich hängt sehr eng mit der Mineralstoffernährung zusammen. Der Stickstoff erscheint hierbei als das wesentlichste Element. Die Wuchsleistung der Fichte auf entkalkten Böden ist grösser als auf Kalkböden. Dies kann allem Anschein nach zum Teil auf eine Verbesserung der Stickstoffernährung zurückgeführt werden. Der Stickstoffspiegel erreicht jedoch auf diesen Standorten - ohne Unterschiede zwischen den verschiedenen Bodentypen und Bodenarten - nur ein mittleres Niveau und erlaubt nicht die Wuchsleistungunterschiede innerhalb dieser Gruppe zu erklären. 
Im Gegensatz dazu ist die Wuchsleistung der Bestände auf Kalkböden sehr eng an die Möglichkeiten der Stickstoffversorgung gebunden. Insbesondere auf den Rendsinen zeigt sich eine deutliche Verbesserung der Stickstoffnadelspiegelwerte; auf Kalkbraunerden welche einen annähernd gleichen Karbonatgehalt jedoch einen wesentlich geringeren Humusgehalt aufweisen, kommt dies nicht sa deutlich zum Ausdruck.

Der auf den Kalkböden beobachtete Produktionsabfall kann jedoch auch teilweise auf einen Manganmangel zurückgeführt werden, da in den kalkreichsten Böden Manganwerte die unter dem Karrenzniveau liegen beobachtet werden konnten. Die statistische Analyse zeigt, dass die Variatonen der Wuchsleistung innerhalb der Kalkböden nicht mit der Manganernährung zusammenhängen. Bei entkalkten Böden besteht ein statistischer Zusammenhang, welcher jedoch keinesfalls kausaler Natur ist.

Auch die Phosphorernährung ist von grosser Bedeutung; sie erlaubt vor allem eine Unterscheidung der Wuchsleistung auf Kalkböden. Hohe Phosphorspiegelwerte können bis zu einem gewissen Grad einen Karbonat - überschuss kompensieren.

Zwischen den Kalziumspiegelwerten und der Wuchsleistung besteht ein negativer Zusammenhang, welcher jedoch nur sehr schwer herausgestellt werden kann. Eine Erhöhung der Kalzium und Kalkwerte im Böden zeigt sich nur sehr schwach in den Kalziumspiegelwerten, da die Kalziumaufnahme gebremst wird. Sie hat jedoch einen grössen Einfluss auf die Sticksteff — und Manganernährung und die reine Kalziumwirkung erscheint dadurch maskiert. Die orthogonale Regressionsanalyse erlaubt einen Teil der Kalziumwirkung zu isolieren; in der progressiven Mehrfachregression wird dieser Effekt von den Nebenwirkungen überdeck.

Abschliessend konnte festgestellt werden, dass die Nadelspiegelwerte von $\mathbf{K}, \mathbf{M g}$, und Fe nur sehr wenig zur Erklärung der Wuchsleistungsunterschiede der Fichte auf diesen Standorten beitragen.

\section{REFERENCES BIBLIOGRAPHIQUES}

Brown J. C., Holmes R. S., Tiffin L. O., 1959. Hypothèses concerning iron chlorosis. Soil Sci. Soc. Amer. Proc., 23, (3), 231-234.

Coic Y., Coppenet M., 1958. Sur la carence en manganèse des céréales dans les sols humifères de Bretagne. Ann. Physiol. vég. suppl. II, III.

Coppenet M., 1959. Le manganèse dans les sols de Bretagne et ses relations avec la croissance des végétaux. Ann. agron., 2, 155-218.

Decourt N., Le Tacon F., 1970. L'épicéa commun (Picéa Excelsa) sur les plateaux calcaires de l'Est de la France. Prévision de la production à l'aide de déterminations pédologiques simples. Ann. sci. forest., (3).

DE Kock P.C., 1955. Iron nutrition of plant at high pH. Soil. Sci., 79, 167-175.

Duchaufour Ph., 1960. Notes sur l'origine de la chlorose de certains conifères sur sol calcaire. Bull. Soc. Bot. Fr., 137, (1-2), 8-12.

Duchaufour Ph., Bonneau M., 1962. E:ude expérimentale de l'influence du calcaire sur la nutrition et la croissance d'un résineux acidiphile. Ann. Ec. Eaux et Forêts, 19, (3), 389-413.

Drouineau G., Gouny P., Mazoyer R., 1950. Influence du calcaire sur la nutrition végétale. Ann. agron., 3', 1-14.

Escouffier Y., 1966. Analyse des composantes principales: utilisation des groupes de variables dans la recherche de la solution. Thèse 3" cycle. Montpellier.

Heller R. Nutrition et métabolisme. 578 p., Masson et $\mathrm{C}^{\mathrm{ie}}$, Paris.

Homes M. V., Van Schoor G. H., 1969. La nutrition minérale des végétaux. Masson et $C^{\text {ie, }}$ Paris.

Jones L. H. P., 1957. The effect of liming a neutral on the uptake of manganese by plants. Plant and Soil, 8, 301-324.

Jones L. H. P., 1957. The effect of liming a neutral soil on the cycle of manganese. Plant and Soil, $8,315-327$. 
Juste C., Pouget R., Bruzau F., 1957. Influence du pH et de l'anion bicarbonique sur l'absorption du fer par des racines de vigne. C.R. Acad. Sci., 264, (24), 2781-4.

Kock P. C. DE, 1955. Iron nutrition of plants at high pH. Soil. Sci., 79, (3), 167-175.

Le Tacon F., Millier C., 1970. La nutrition minérale de l'épicéa commun (Picea excelsa link.) en sols carbonatés et en sols décarbonatés. Essai sur le comportement du calcium et du manganèse. Ann. Sci. forest., (1), 1-24.

Le Tacon F., Oswald H., Tomassone R., 1970. La nutrition minérale de l'Epicéa en haute Ardèche. Liaison avec la production. Ann. sci. forest., (sous presse).

Page E. R., 1962. Studies in soil and plant manganese. The relationship of soil $\mathrm{pH}$ to manganese availability. Plant and soil, 16, (2), 247-257.

Rich C. I., 1956. Manganese content of peanut leaves as related to soil factors. Soil. Sci., 82, (5), 353-363.

SEAL H., 1964. Multivariate statistical analysis for biologists. Methuen, Londres.

Sutcliffe D. SC., 1962. Mineral salts absorption in plants. Pergamon press. Oxford, London, New York, Paris.

Tomassone R., 1967. Une méthode d'investigation. La régression orthogonale. Ann. Sci. forest., 24, (3), p. 233-258.

Tomassone R., 1967. Régression multiple progressive. Station de Biométrie. C.N.R.F. Programme FORTRAN 67.009. 
ANNEXe

Niveau de nutrition de l'ensemble des placettes (Moyenne de 5 échantillons)

Mineral nutrition characteristics of all plots (mean of five samples)

\begin{tabular}{|c|c|c|c|c|c|c|c|c|c|c|}
\hline $\begin{array}{l}\text { Sous- } \\
\text { ensemble } \\
\text { de sol }\end{array}$ & $\begin{array}{c}\mathrm{N}^{\circ} \text { pla- } \\
\text { cette }\end{array}$ & Age & $\begin{array}{l}\text { Hauteur } \\
\text { dominante } \\
\text { en } m\end{array}$ & $\mathrm{~N} \%$ & $\mathrm{P}_{2} \mathrm{O}_{5} \%$ & $\mathrm{~K} \%$ & $\mathrm{Ca} \%$ & $\mathrm{Mg} \%$ & Mn / / & $\mathrm{Fe} / / c$ \\
\hline 11 & $\begin{array}{l}20 \\
21 \\
22 \\
37 \\
41 \\
42 \\
55 \\
58 \\
85\end{array}$ & $\begin{array}{r}34 \\
36 \\
38 \\
45 \\
32 \\
35 \\
18 \\
104 \\
36\end{array}$ & $\begin{array}{r}6,44 \\
8,31 \\
16,81 \\
15,31 \\
5,60 \\
11,16 \\
4,02 \\
29,60 \\
13,58\end{array}$ & $\begin{array}{l}0,68 \\
0,77 \\
1,25 \\
1,04 \\
1,03 \\
1,25 \\
0,63 \\
1,14 \\
1,14\end{array}$ & $\begin{array}{l}0,28 \\
0,35 \\
0,39 \\
0,45 \\
0,29 \\
0,37 \\
0,22 \\
0,33 \\
0,38\end{array}$ & $\begin{array}{l}0,54 \\
0,59 \\
0,54 \\
0,60 \\
0,75 \\
0,81 \\
0,76 \\
0,76 \\
0,68\end{array}$ & $\begin{array}{l}1,17 \\
1,30 \\
1,02 \\
0,84 \\
0,76 \\
0,59 \\
0,94 \\
0,98 \\
0,97\end{array}$ & $\begin{array}{l}0,06 \\
0,08 \\
0,08 \\
0,13 \\
0,07 \\
0,14 \\
0,09 \\
0,09 \\
0,17\end{array}$ & $\begin{array}{l}0,07 \\
0,06 \\
0,07 \\
0,06 \\
0,04 \\
0,02 \\
0,10 \\
0,07 \\
0,04\end{array}$ & $\begin{array}{l}0,12 \\
0,10 \\
0,13 \\
0,11 \\
0,10 \\
0,13 \\
0,12 \\
0,09 \\
0,10\end{array}$ \\
\hline 12 & $\begin{array}{l}02 \\
08 \\
14 \\
43 \\
46 \\
59 \\
69 \\
73 \\
80 \\
81 \\
82 \\
83 \\
84\end{array}$ & $\begin{array}{l}91 \\
37 \\
56 \\
37 \\
54 \\
75 \\
51 \\
44 \\
60 \\
37 \\
56 \\
56 \\
57\end{array}$ & $\begin{array}{l}24,34 \\
17,35 \\
17,55 \\
16,90 \\
18,75 \\
28,85 \\
23,50 \\
16,33 \\
22,58 \\
13,46 \\
20,29 \\
19,52 \\
16,40\end{array}$ & $\begin{array}{l}1,11 \\
1,64 \\
1,41 \\
1,44 \\
1,20 \\
1,45 \\
1,39 \\
1,07 \\
1,18 \\
1,18 \\
1,36 \\
1,28 \\
1,42\end{array}$ & $\begin{array}{l}0,23 \\
0,35 \\
0,28 \\
0,41 \\
0,32 \\
0,36 \\
0,43 \\
0,26 \\
0,38 \\
0,36 \\
0,37 \\
0,30 \\
0,38\end{array}$ & $\begin{array}{l}0,56 \\
0,67 \\
0,69 \\
0,75 \\
0,69 \\
0,78 \\
0,73 \\
0,74 \\
0,76 \\
0,66 \\
0,65 \\
0,57 \\
0,85\end{array}$ & $\begin{array}{l}1,02 \\
0,95 \\
0,79 \\
0,87 \\
0,69 \\
0,80 \\
0,61 \\
0,82 \\
0,92 \\
1,18 \\
1,05 \\
0,80 \\
0,96\end{array}$ & $\begin{array}{l}0,06 \\
0,05 \\
0,10 \\
0,14 \\
0,18 \\
0,08 \\
0,11 \\
0,14 \\
0,13 \\
0,15 \\
0,05 \\
0,08 \\
0,16\end{array}$ & $\begin{array}{l}0,11 \\
0,12 \\
0,11 \\
0,01 \\
0,09 \\
0,20 \\
0,11 \\
0,19 \\
0,03 \\
0,02 \\
0,05 \\
0.16 \\
0,05\end{array}$ & $\begin{array}{l}0,11 \\
0,13 \\
0,07 \\
0,12 \\
0,04 \\
0,07 \\
0,10 \\
0,35 \\
0,11 \\
0,09 \\
0,12 \\
0,16 \\
0,10\end{array}$ \\
\hline 13 & $\begin{array}{l}16 \\
17 \\
18 \\
19 \\
27 \\
28 \\
29 \\
38 \\
39 \\
40 \\
40 \\
45 \\
54 \\
79\end{array}$ & $\begin{array}{l}78 \\
74 \\
73 \\
75 \\
48 \\
50 \\
49 \\
54 \\
53 \\
50 \\
54 \\
50 \\
78 \\
60\end{array}$ & $\begin{array}{l}22,35 \\
22,30 \\
20,18 \\
20,89 \\
22,30 \\
20,47 \\
19,88 \\
20,77 \\
15,10 \\
20,79 \\
20,90 \\
20,24 \\
28,42 \\
20,00\end{array}$ & $\begin{array}{l}1,34 \\
1,26 \\
1,14 \\
1,28 \\
1,35 \\
1,43 \\
1,52 \\
1,23 \\
1,29 \\
1,21 \\
1,42 \\
1,40 \\
1,18 \\
1,47\end{array}$ & $\begin{array}{l}0,21 \\
0,29 \\
0,19 \\
0,23 \\
0,29 \\
0,34 \\
0,37 \\
0,39 \\
0,45 \\
0,41 \\
0,32 \\
0,51 \\
0,34 \\
0,41\end{array}$ & $\begin{array}{l}0,49 \\
0,61 \\
0,56 \\
0,47 \\
0,38 \\
0,42 \\
0,64 \\
0,63 \\
0,55 \\
0,72 \\
0,66 \\
0,48 \\
0,67 \\
0,68\end{array}$ & $\begin{array}{l}1,06 \\
0,74 \\
0,92 \\
0,99 \\
1,03 \\
0,95 \\
0,90 \\
0,69 \\
0,72 \\
0,71 \\
0,78 \\
0,73 \\
0,58 \\
0,62\end{array}$ & $\begin{array}{l}0,10 \\
0,12 \\
0,09 \\
0,08 \\
0,14 \\
0,13 \\
0,16 \\
0,14 \\
0,09 \\
0,08 \\
0,10 \\
0,12 \\
0,06 \\
0,10\end{array}$ & $\begin{array}{l}0,06 \\
0,03 \\
0,06 \\
0,05 \\
0,06 \\
0,18 \\
0,06 \\
0,04 \\
0,06 \\
0,02 \\
0,09 \\
0,13 \\
0,02 \\
1,33\end{array}$ & $\begin{array}{l}0,05 \\
0,06 \\
0,06 \\
0,20 \\
0,18 \\
0,24 \\
0,19 \\
0,12 \\
0,12 \\
0,14 \\
0,07 \\
0,03 \\
0,10 \\
0,30\end{array}$ \\
\hline
\end{tabular}




\begin{tabular}{|c|c|}
\hline 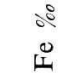 & 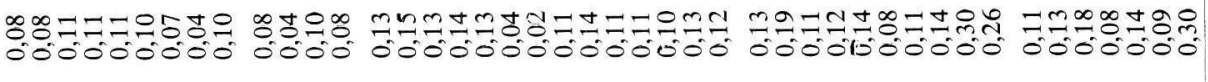 \\
\hline$\sum^{8}$ & 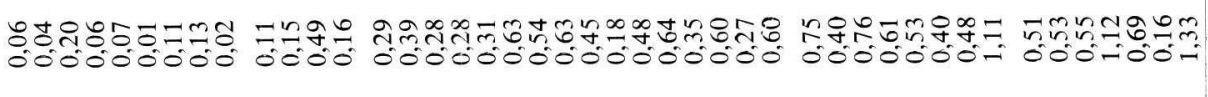 \\
\hline$\sum^{\infty}$ & 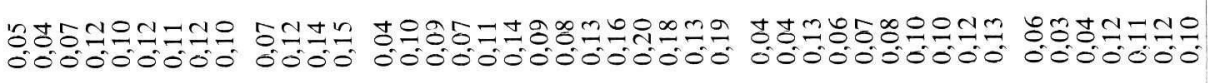 \\
\hline ن̃ & 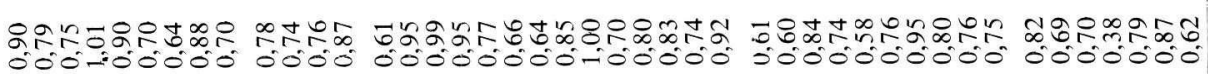 \\
\hline 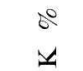 & 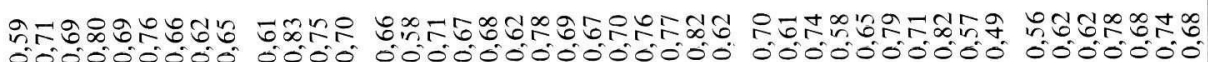 \\
\hline 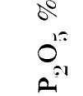 & 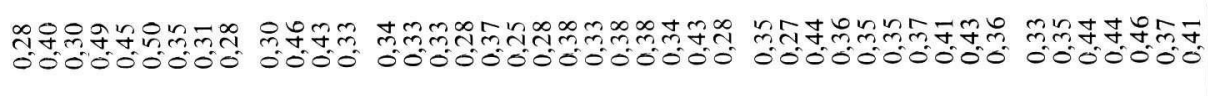 \\
\hline Z & 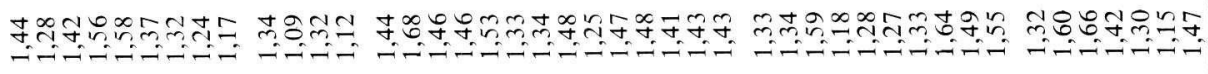 \\
\hline 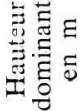 & 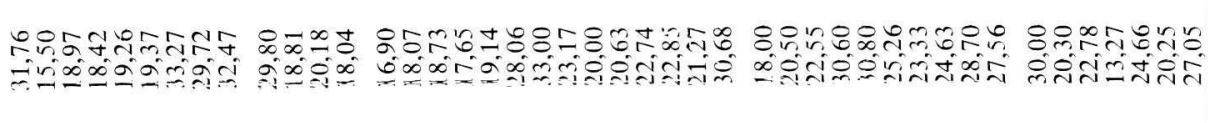 \\
\hline$\stackrel{\substack{\infty \\
\&}}{\infty}$ & 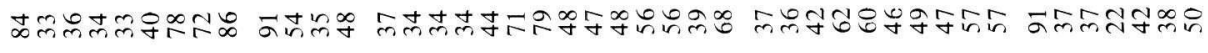 \\
\hline 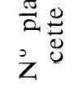 & 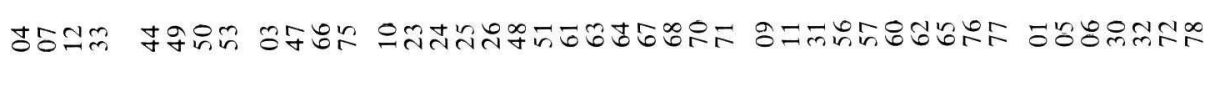 \\
\hline 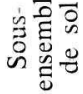 & \pm \\
\hline
\end{tabular}

